Check for updates

Cite this: J. Mater. Chem. B, 2021, 9, 5025

\section{Engineering next-generation bioinks with nanoparticles: moving from reinforcement fillers to multifunctional nanoelements}

\author{
Syeda M. Bakht, $\uparrow^{\mathrm{ab}}$ Alberto Pardo, $\uparrow^{\mathrm{abc}}$ Manuel Gómez-Florit, ${ }^{\mathrm{ab}}$ Rui L. Reis, ${ }^{\mathrm{ab}}$ \\ Rui M. A. Domingues*ab and Manuela E. Gomes (iD *ab
}

\begin{abstract}
The application of additive manufacturing in the biomedical field has become a hot topic in the last decade owing to its potential to provide personalized solutions for patients. Different bioinks have been designed trying to obtain a unique concoction that addresses all the needs for tissue engineering and drug delivery purposes, among others. Despite the remarkable progress made, the development of suitable bioinks which combine printability, cytocompatibility, and biofunctionality is still a challenge. In this sense, the well-established synthetic and functionalization routes to prepare nanoparticles with different functionalities make them excellent candidates to be combined with polymeric systems in order to generate suitable multi-functional bioinks. In this review, we briefly discuss the most recent advances in the design of functional nanocomposite hydrogels considering their already evaluated or potential use as bioinks. The scientific development over the last few years is reviewed, focusing the discussion on the wide range of functionalities that can be incorporated into 3D bioprinted constructs through the addition of multifunctional nanoparticles in order to increase their regenerative potential in the field of tissue engineering.
\end{abstract}

Received 31st March 2021 Accepted 14th May 2021

DOI: 10.1039/d1tb00717c

rsc.li/materials-b is the design of the suitable bioinks, that is, the biomaterials that combined with cells and other elements, can be precisely distributed hierarchically and spatially according to the desired shape. ${ }^{5,6}$ In many cases, the cytocompatible printing conditions for cells (bioprinting window) are not the same that allow highresolution degrees on the designed composites, hindering the preparation of personalized constructs. ${ }^{7}$

The most common bioinks for TE purposes are based on hydrogels. These are characterized by water-rich networks similar to those observed in native tissues, as well as by high biocompatibility and tunable mechanical performance. ${ }^{8-11}$ However, the high polymer concentration and crosslinking density required to obtain printable hydrogels limit the spreading, migration and/or differentiation of the encapsulated cells. Moreover, using single hydrogel-based bioink formulations it's hardly enough to design 3D bioprinted scaffolds that properly mimic the specific structure and properties of most tissues. In this sense, printable hydrogels can be modified with nanoparticles (NPs) and/or biological factors, among others, for the biofabrication of $3 \mathrm{D}$ constructs that seek to replicate the main characteristics and properties of native tissues. ${ }^{12-15}$

In pioneering works that have used such strategy, the addition of nanofillers was shown to allow tuning the physical characteristics of the bioinks, such as viscosity, stiffness, and nonlinear viscoelasticity (shear-thinning behavior), in order to 
improve their printability. ${ }^{16,17}$ In general, the mechanical properties of hydrogels proportionally improve with nanofillers concentration, but the incorporation of these nanostructures can, on the other hand, have either positive or negative affects over the printing fidelity of the constructs, depending on the type of filler and its concentration. ${ }^{17}$ High concentrations of inorganic NPs strongly increase the bioink viscosity, requiring higher printing pressures, which, at the same time, decrease cell viability. Thus, to remain within the biofabrication window, ${ }^{18}$ the bioinks with inorganic NPs typically compromise printing fidelity to maintain high cell viability. For instance, synthetic nanosilicateclays ${ }^{19}$ or silica $\mathrm{NPs}^{20}$ were established as efficient nanofillers to provide bioinks with easier printability and promote the adhesion and proliferation of the cells encapsulated in the 3D bioprinted constructs.

The most recent advances in the design of complex bioinks have shown that the fillers added to printable systems do not only actuate as reinforcement elements but can also provide the inks with essential biofunctionalities that can be exploited in the field of TE of specific tissues. In fact, each tissue of the human body is characterized by unique physicochemical characteristics that regulate cell fate and confers its specific properties. ${ }^{21}$ For instance, tendon or skeletal muscle tissues are characterized by highly-anisotropic uniaxial aligned topography that confers cells elongated morphologies, ${ }^{22,23}$ while bone tissue presents high stiffness as one of their most remarkable characteristics. $^{24}$ In this way, the use of different nanofillers such as stimuli-responsive NPs, ceramic NPs, or nanomaterials incorporating biological factors, has been proposed to add functionality to the bioinks in order to mimic the requirements of specific tissues and complex cells microenvironments in the designed 3D-bioprinted composites. ${ }^{25-27}$ Overall, the incorporation of nanofillers in hydrogel-based bioinks has not only been evaluated to improve the combination of printability and cytocompatibility of the $3 \mathrm{D}$ bioprinted composites but also to provide them with the functional properties required to control cells fate and reproduce the physicochemical microenvironment of different native tissues.

In this review, we briefly discuss the main strategies based on the addition of nanofillers to provide hydrogel-based biomaterials with different functionalities that improve their bioactivity and regenerative potential, targeting their use of in 3D bioprinting technology. In this way, the most recent works that explored the incorporation of stimuli-responsive NPs to design 3D bioprinted constructs with anisotropic magnetic or electric properties are reviewed, as well as those which evaluated the mineralization effect derived from the addition of inorganic particles. The modification of bioinks using nanomaterials decorated with biological factors that induce biochemical signals to control cell fate and the incorporation of NPs for real-time monitoring are also discussed (Fig. 1). In each of these approaches, the essential characteristics/properties that the incorporated nanofillers must meet to provide bioinks with the desired functionality are commented while considering as well the potential applications of the 3D bioprinted functional nanocomposites in specific areas of $\mathrm{TE}$ and regenerative medicine.

\section{Nanocomposite bioinks}

Among different possibilities to enhance the performance of hydrogel-based bioinks, the incorporation of micro-and nanofillers is one of the most efficient approaches to provide the $3 \mathrm{D}$ bioprinted composites with the functional properties required for TE purposes. By thoroughly understanding the precise requirements of the target tissues/organs and the versatile response of the incorporated organic and/or inorganic NPs, the nanocomposite bioinks can be tuned to develop 3D-printed scaffolds with the desired characteristics. In this sense, the nanofillers not only passively improve the printability and shape fidelity of the bioprinted constructs, but can also actively contribute to meet the desired outcomes of the fabricated scaffolds. This can include either to influence cell proliferation or differentiation into the intended cell type, to maintain/ provide a cell niche by emulating the microenvironment of the tissue/organ of interest, and/or to enable real-time monitoring of the cellular processes during the maturation. Thus, for instance, the addition of ceramic NPs has been positively tested to increase the toughness and the stiffness of the scaffolds for bone $\mathrm{TE},{ }^{28}$ while electrically-responsive NPs such as carbon nanotubes were used to provide hydrogels with the electrical properties required to engineer skeletal muscle, neural or cardiac tissues. ${ }^{29}$

In this section, we briefly reviewed the main strategies explored to prepare nanocomposite bioinks for biomedical purposes, classifying them into four groups according to the nature and the characteristics of the incorporated nanomaterials: biological factors-conjugated NPs, ceramic NPs, stimuli-responsive NPs, and NPs for real-time monitoring (Fig. 2).

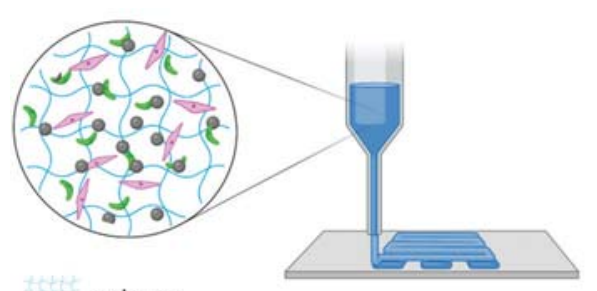

polymer

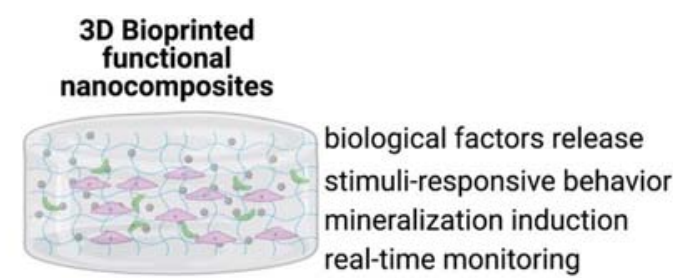

functional nanofillers

Fig. 1 Schematic illustration: preparation of 3D bioprinted constructs incorporating nanofillers with multiple functionalities. 


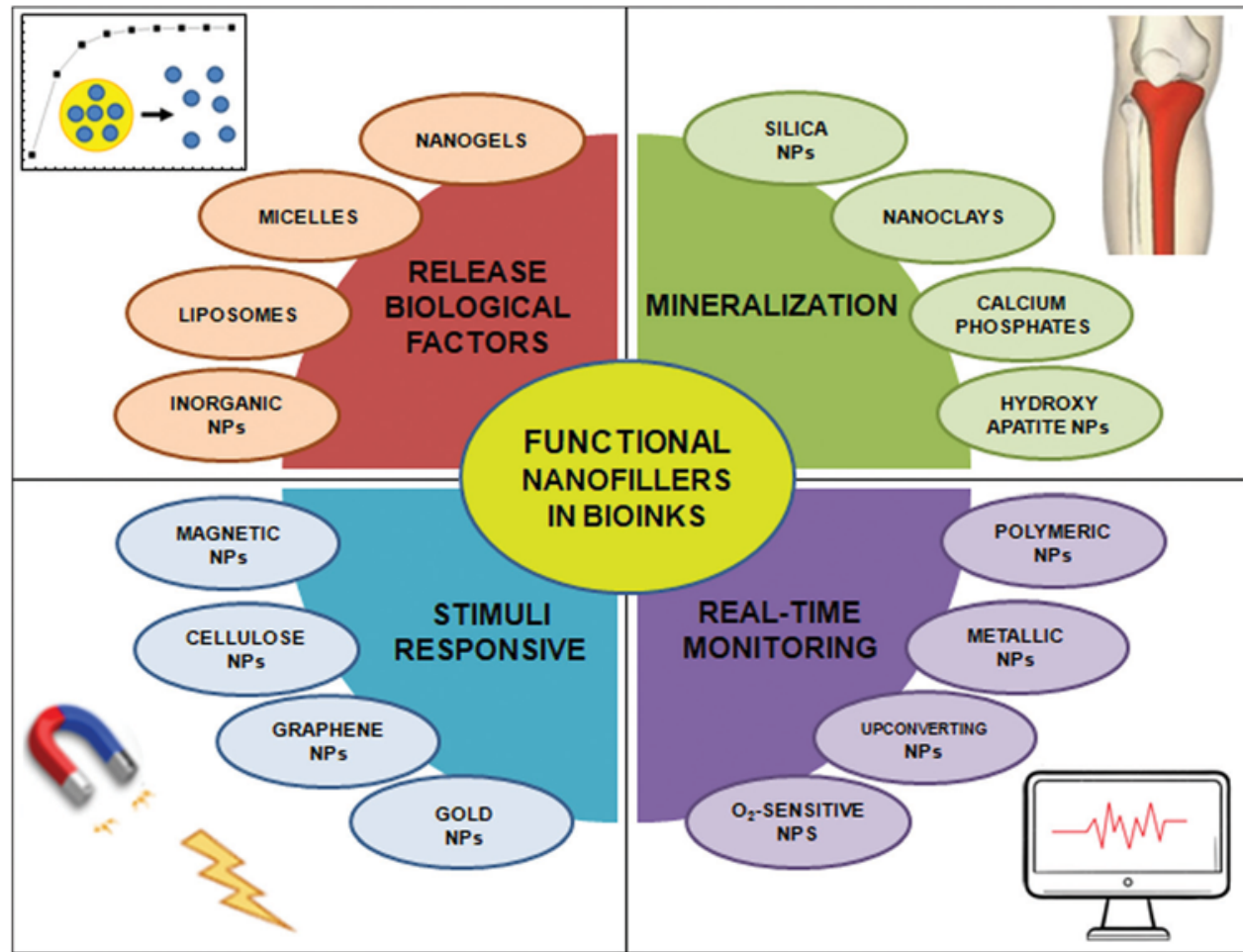

Fig. 2 Classification of some representative nanofillers that can be incorporated within bioinks to provide them with different functionalities: release of biological factors, enhancement of mineralization degree, stimuli-responsive capability and real-time monitoring properties.

\subsection{Bioinks with nanoparticles for controlled release of drugs and bioactive molecules}

Human organs are composed of multiple tissues organized into complex architectures and gradients that cooperatively contribute to their specific functionality. Tissue homeostasis and its regeneration after injury are controlled by a finely orchestrated biological signaling that involves different biophysical and biochemical cues. Our capacity to develop successful regenerative strategies depends on the ability to replicate the characteristic tissue spatiotemporal signal transduction pathways, transcription factor instructions and protein regulation. ${ }^{30}$

Different types of organic and inorganic NPs (e.g. liposomes, dendrimers, polymeric micelles, nanogels, or silica nanomaterials) have been extensively used as carrier vehicles for the controlled delivery of bioactive molecules of interest for regenerative therapies, including cytokines, chemokines, and growth factors, ${ }^{30}$ or for the delivery of genetic material (e.g. plasmid DNA or siRNA). ${ }^{31-34}$ Nanocarriers can be engineered not only to protect their sensitive cargo from harsh biomaterial processing conditions or from deactivation within biological environments but also to enable its programmed release triggered by specific stimuli. ${ }^{35}$ This set of unique characteristics has made them a valuable tool as depots of signaling molecules in the development of smart hydrogel systems with spatial- and temporal-controlled release of chemical cues for TE applications. ${ }^{33,36}$ Beyond the possibility of participating in the crosslinked network to improve hydrogels mechanical properties, the major advantage of incorporating drug-loaded NPs into polymer networks is that the drug-loading process and hydrogel preparation become relatively independent processes, increasing, therefore, the engineering liberty to separately tune the drug release kinetics and the hydrogel physicochemical properties. As a representative example of such systems, mesoporous silica NPs loaded with bone-forming peptide- 1 were incorporated into alginate-based hydrogels (RGD modified). ${ }^{37}$ This hybrid hydrogel resulted in an engineered time-responsive osteogenic niche with the ability to sequentially trigger stem cell adhesion, proliferation, and osteogenic differentiation. In a different approach, hybrid hydrogels prepared by crosslinking of pullulan-based nanogels encapsulating multiple GFs (human bone morphogenetic protein 2 (BMP-2) and human fibroblast growth factor 18 (FGF18)) with thiol-modified polyethylene glycol, generated a biodegradable hydrogel that promoted effective bone repair in critical-size skull bone defects. ${ }^{38}$

The potential of 3D bioprinting technology to fabricate cellularized constructs with complex architectures, tailorable mechanical properties, and spatially controlled biological function would greatly benefit from adopting hybrid hydrogels incorporating drug-releasing nanocarriers on their bioink development concepts. Such strategies would significantly contribute to increasing the biofunctionality of current hydrogel-based bioinks, allowing to more closely recreating the cascades of biological signaling that may improve the regenerative outcomes of fabricated constructs. This would be particularly relevant when targeting tissue interfaces that show cellular and ECM gradients with different spatiotemporal biological signaling requirements, such as osteochondral or tendon-to-bone tissues. ${ }^{39}$ These and many other living tissues 
with spatial-dependent properties and cellular composition could thus be simulated, for instance, by printing gradients or interfacing different bioinks containing nanocarriers with the relevant bioactive molecules of interest.

Interestingly, this strategy is making its way into the design concepts of several 3D printed scaffolds engineered for the regeneration of complex tissues. ${ }^{40,41}$ For instance, the sustained and spatially controlled release of tenogenic (CTGF), chondrogenic (TGF- $\beta 3$ + CTGF), and osteogenic (BMP-2) growth factors from microcarriers embedded in 3D printed scaffolds induced stem cell differentiation gradients that better recreate the phenotypes existing at tendon-to-bone interphases. ${ }^{42}$ A similar strategy was adopted to fabricate disc scaffolds with micro-precise spatiotemporal delivery of CTGF and TGF- $\beta 3$, mimicking the native multiphase fibrocartilage of temporomandibular joint (TMJ). ${ }^{41}$

However, so far, only a few bioink hydrogels incorporating drug-release nano/microcarriers have been reported. For instance, the sustained release of VEGF from gelatin microparticles was used to create defined pro-vasculogenic differentiation regions in $3 \mathrm{D}$ bioprinted heterogeneous constructs that led to a significant increase in its vascularization when applied in vivo (Fig. 3a). ${ }^{43}$
Similarly, the sustained release of TGF- $\beta 1$ from nanospheres embedded in constructs fabricated through stereolithography $3 \mathrm{D}$ bioprinting with methacrylated gelatin (GelMA)/polyethylene glycol diacrylate (PEGDA) based bioinks improved the chondrogenic differentiation of encapsulated mesenchymal stem cells (MSCs) (Fig. 3b). ${ }^{44}$ In a different recently proposed approach, geneactivated bioinks were developed by the incorporation of amphipathic peptide-plasmid DNA complexes for the non-viral transfer of either osteogenic (BMP-2) or chondrogenic (combination of TGF- $\beta 3$, BMP-2, and SOX9) genes to encapsulated stem cells. ${ }^{45}$ Remarkably, this concept enabled spatially direct stem cell differentiation in a bilayered construct for the recapitulation of zonal phenotypes of the osteochondral unit, demonstrating the potential of these gene-activated bioinks to engineer spatially complex tissues.

Although still in its infancy, these examples serve to demonstrate that the incorporation of drug-releasing nanocarriers into bioinks can offer a valuable biomaterial design route to address the need for increasing the biological functionality of current bioinks. These strategies will certainly contribute to better recapitulate the complexity of biological signaling existing in

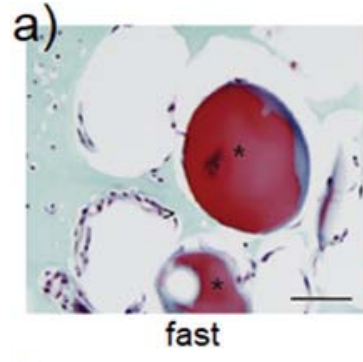

b)

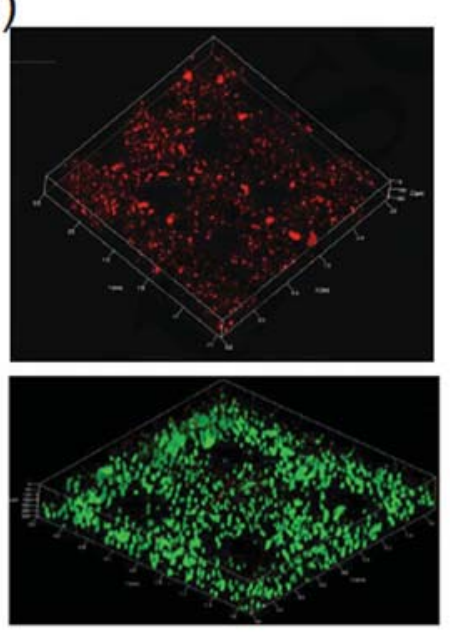

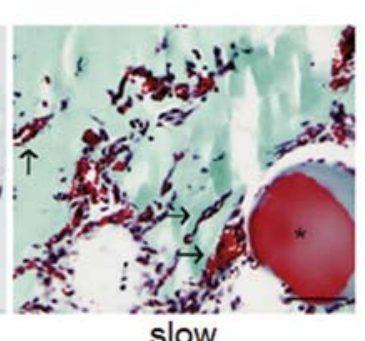

slow

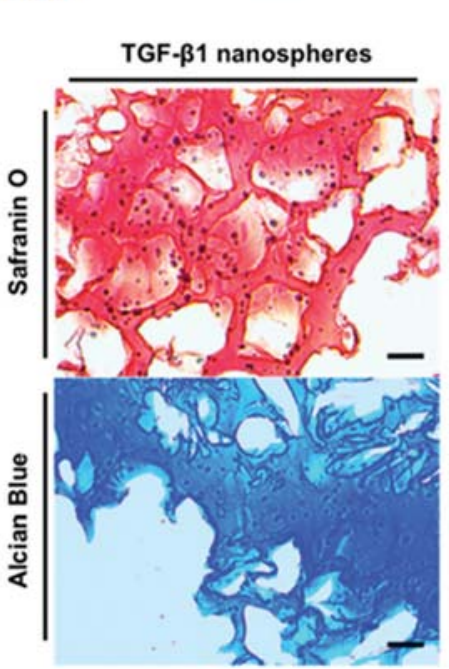

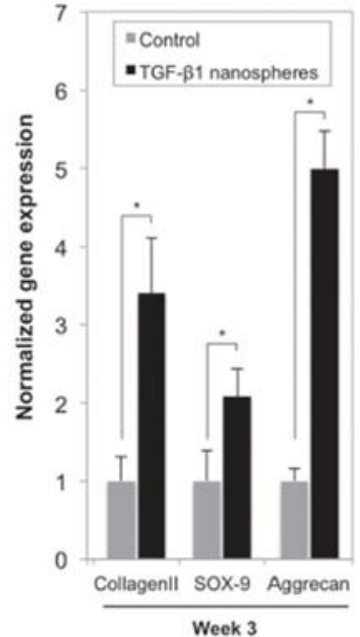

Fig. 3 Bioinks with biological factors-conjugated nanoparticles: (a) quantification of vessel formation using Goldner's trichrome staining in hEPCs seeded scaffolds after one week of subcutaneous implantation in mice. The constructs with VEGF-loaded gelatin particles (slow VEGF release) presented a higher vascularization than those in which free VEGF was directly incorporated (fast VEGF release) (scale bars $=50 \mu \mathrm{m}$ ). 3D bioprinted heterogeneous scaffolds with two different regions were also prepared (- without VEGF, + VEGF-laden particles), observing no vessels formation in the control part of the scaffolds and well-defied perfused vessels in the region with slow release of VEGF (reproduced with permission from ref. 43 copyright 2014 Elsevier). (b) Fluorescence microscopy images of TGF- $\beta 1$-embbedded nanospheres (in red) and human bone marrow MSCs (in green) incorporated in 3D printed constructs. After three weeks of chondrogenic induction, the presence of TGF- $\beta 1$-loaded NPs induced chondrogenic ECM secretions (the sections were stained with safranin $O$ and alcian blue to bind to cartilage proteoglycans and glycosaminoglycans, respectively) and high expressions levels of chondrogenesis-related genes SOX-9, Collagen II and Aggrecan (reproduced with permission from ref. 44 copyright 2014 IOP Science). 
living tissues and consequently the potential of success of 3D bioprinted constructs.

\subsection{Enhancing mineralization using biofunctional inorganic nanoparticles}

Conventional hydrogels are able to mimic different characteristics of the ECM of native tissues although they are typically associated with a number of disadvantages such as their low strength and poor mineralization upon implantation, which limits their application for engineering load-bearing tissues such as bone and cartilage. ${ }^{46,47}$ As mentioned before, over the past years, different types of NPs have been extensively used as mechanical reinforcers of hydrogels and bioinks. ${ }^{13,48,49}$ Inspired from the nanomaterials found in biological tissues, a range of bioactive inorganic NPs, including calcium phosphates (hydroxyapatite, tricalcium phosphates) and nanosilicates (silica NPs, bioglass NPs, and nanoclays), has been explored to develop bioinks with adequate rheological characteristics to obtain 3D constructs showing high printing fidelity and biocompatibility. ${ }^{50-52}$ Nonetheless, the mentioned inorganic NPs own more functionalities beyond simple mechanical reinforcement since most of them consist of minerals that are present in human tissues and are necessary for normal physiology (e.g., around $60 \mathrm{wt} \%$ of bone is composed of hydroxyapatite). ${ }^{53}$ Moreover, these type of NPs has shown good biocompatibility and favorable biological responses in different cell types. ${ }^{54,55}$ For example, calcium and phosphate, which can be released from calcium phosphates, stimulate the deposition of the mineralized matrix by osteoblasts and prevent bone loss. ${ }^{56}$ Similarly, silicon, another mineral component present in nanosilicates, stimulates the osteogenic differentiation of human stem cells and also promotes collagen type I synthesis. ${ }^{57}$ The release of these minerals from inorganic NPs can be leveraged to enhance the bioactivity of bioinks and improve cell adhesion, proliferation and influence differentiation of diverse cell types into specific lineages (e.g. osteogenic).

Various nanocomposite bioinks based on calcium phosphate nanoelements have been developed to enhance osteogenic differentiation and to promote mineralization in tissue-engineered 3D-bioprinted constructs. For example, in a recent study, human adipose-derived stem cells (hASCs) encapsulated in chitosanbased bioinks incorporating $2 \% \mathrm{w} / \mathrm{v}$ nanohydroxyapatite particles showed peak expression levels for early and late stages osteogenic markers. ${ }^{58}$ It was concluded that cells within the nanocomposite hydrogels had mineralized and differentiated into osteogenic lineage after 21 days of culture. Likewise, micro-and nanohydroxyapatite incorporated within collagen, gelatin, and hyaluronic acid-based bioinks promoted the mineralization of the 3D bioprinted constructs. ${ }^{59-61}$

Nanosilicates are a different type of inorganic nanofillers with anisotropic platelet-shaped morphology $(20-50 \mathrm{~nm}$ in diameter and 1-2 nm thickness) showing a high degree of functionality and applications in cosmetics and toothpaste as well as drug delivery and TE. ${ }^{62,63}$ Among these, LAPONITE ${ }^{\mathbb{R}}$ XLG has been extensively studied for biomedical applications due to its lower heavy metal content. $^{51}$ The use of silicate nanoplatelets was shown to significantly enhance the osteogenic differentiation of human mesenchymal stem cells (hMSCs) in typical 2D culture systems ${ }^{64,65}$ and encapsulated in GelMA hydrogels using growth factors free medium. ${ }^{66}$ Moreover, these NPs show negatively charged faces and positively charged edges, which allow reversible electrostatic interactions with charged polymer backbones. Relying on this characteristic, Gaharwar et al. used nanosilicates to reinforce an ionic-covalent entanglement hydrogel composed of GelMA and $\kappa$-carrageenan, creating a dually reinforced hydrogel network. ${ }^{67}$ This nanoengineered ionic covalent entanglement (NICE) bioink showed an excellent printability and allowed to print 3D constructs with high aspect ratio and high printing fidelity while, at the same time, showing high cell viability and proliferative capacity. More notably, in a later work, the bioprinted constructs induced endochondral differentiation of encapsulated hMSCs in the absence of osteoinductive agents (Fig. 4). ${ }^{68,69}$ Besides, due to their shape, these anisotropic NPs are able to align under shear during 3D printing. ${ }^{67,68}$ Therefore, the incorporation of anisotropic fillers can be leveraged for supporting not only the printing process but also to promote cell alignment required to recreate anisotropic tissues such as tendon, muscle, cartilage, and their interfaces. ${ }^{12,21}$

Other types of nanofillers have also been evaluated to provide bioinks with enhanced mineralization functionality. For instance, the addition of strontium-carbonate NPs has been demonstrated as an efficient strategy to increase the printability and the mineralization degree of GelMA-based hydrogels, thus inducing the osteogenic differentiation of the MSCs encapsulated within the nanocomposite 3D bioprinted constructs. ${ }^{70}$

Although inorganic NPs have been mostly used to develop bioinks for bone regeneration approaches, their beneficial effects in cells from softer tissues (e.g. cartilage and osteochondral tissues) have widened the application of bioinks containing these NPs. ${ }^{50,51}$ In addition, considering the capabilities of bioprinting to place cells and materials in the 3D space, the recreation of gradient tissue interfaces (tendon-to-bone, osteochondral tissues) can largely benefit from these approaches. Moreover, as discussed in previous sections, inorganic NPs have also been used as therapeutic drug/growth factors delivery agents, which could potentially lead to the development of single NPs showing multiple functionalities.

\subsection{Providing stimuli-responsive functionalities with smart nanoparticles}

The design of hydrogels with stimuli-responsive properties is an essential challenge for their subsequent application in different areas of the biomedical field. Actually, these hydrogels have been shown to control the release in drug delivery formulations or to mimic the native tissues in terms of physicochemical properties and/or control over the behavior of the encapsulated cells for TE purposes. ${ }^{71,72}$ The chemical modification of the hydrogels precursor solutions to render them sensitive to light, acoustic, or $\mathrm{pH}$ stimuli has been widely analyzed, being the incorporation of nano-structured smart materials that provide hydrogels with responsive properties another well-explored strategy. ${ }^{73,74}$ So far, NPs with magnetic and electric properties have been the most evaluated as potential nanofillers in the design of stimuliresponsive nanocomposite hydrogels for biomedical applications. 
a)
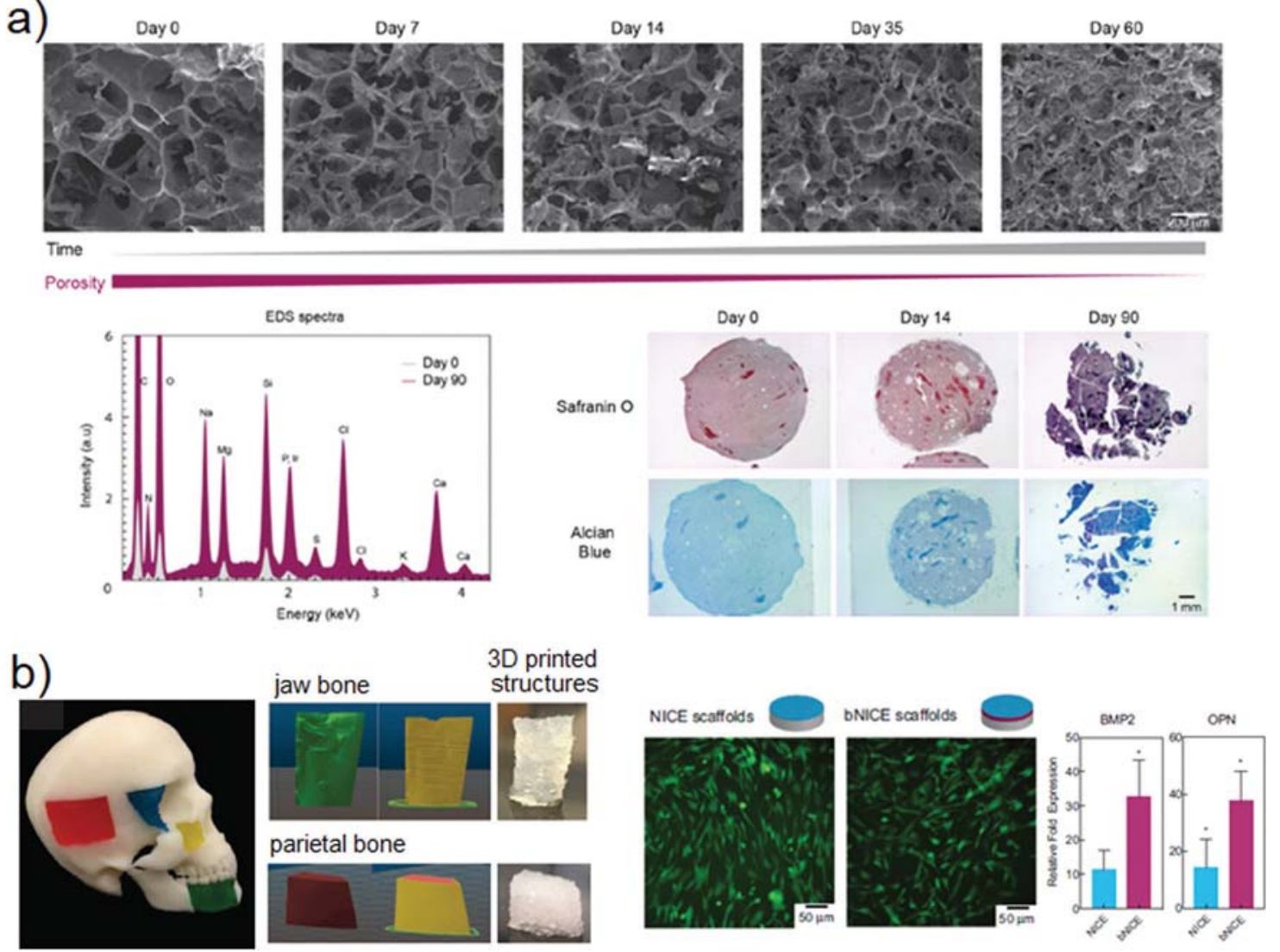

Fig. 4 Bioinks with ceramic nanoparticles to induce mineralization: (a) development of NICE bioink composed of GelMA, kappa-carrageenan and LAPONITE ${ }^{\mathbb{R}}$ XLG, loaded with MSCs. Scanning electron microscopy images showed a gradual decrease in the pore size of cell-laden 3D bioprinted scaffolds due to deposition of ECM by cells. EDS analysis corroborated an increase in calcium and phosphates content after 60 days of culture due to osteogenic differentiation of hMSCs, while histology stains demonstrated the osteochondral production of mineralized cartilage ECM (Safranin O stains cartilage tissue in varying shades of red, while bone tissue is bluish-purple. Alcian Blue stains connective tissue light blue and cartilage dark blue) (reproduced with permission from ref. 68 copyright 2020 American Chemical Society). (b) In a more recent work from the same group, NICE bioink was used to reproduce various anatomical defects, such as jaw bone, eye socket or parietal bone, with high printing fidelity. Moreover, the osteoinductive properties of 3D bioprinted NICE constructs can be augmented by depositing a pluripotent stem cell-derived ECM on them (bNICE: bioconditioned NICE scaffolds). bNICE scaffolds increased the viability of the encapsulated hMSCs and the expression of osteogenic markers BMP-2 and OPN after 2 and 21 days of culture, respectively (reproduced with permission from ref. 69 copyright 2020 Wiley-VCH).

In this sense, the development of scaffolds with electricresponsive properties is an essential challenge in fields such as neural, muscle, or cardiac TE, where the transmission of nerve impulses across the body and heart contractile properties present electrical origins. Although the use of polymers with weak conductive properties like polypyrrole or polyaniline has been evaluated for these applications, ${ }^{75,76}$ the incorporation of electric nanofillers based on carbon structures (e.g. carbon nanotubes and graphite-based nanomaterials) or noble metals (e.g. gold and silver NPs) has been a more widely analyzed strategy. ${ }^{77-80}$ On the other hand, magnetic materials have been used to provide nanocomposite hydrogels with anisotropic structure and magnetomechanic stimulation properties through the application of external magnetic fields. ${ }^{12}$ The intrinsic properties of magnetic nanoparticles (MNPs) allow control over their distribution within the 3D space of hydrogel networks by applying magnetic radiations, opening the possibility to the design of anisotropic magneticallyresponsive constructs. ${ }^{12}$ MNPs with superparamagnetic behavior are especially appealing for this purpose since in this regime, the particles do not retain any magnetization energy after the application of an external magnetic field, which precludes the formation of aggregates and allows full control over their magnetic guidance/distribution. ${ }^{81,82}$

The incorporation of nanofillers to render light-responsive hydrogels has also been explored, being graphite-graphene oxide structures among the most commonly utilized for this fabrication strategy. ${ }^{83,84}$ Besides their aforementioned electrical response, graphite-derived composites are also light-sensitive, which allows their use as reinforcement materials to develop hydrogels with adjustable properties through the application of external light stimuli. ${ }^{85}$ The infrared light absorption properties of graphite oxide nanostructures were used, for instance, to endow faster photothermal excited responsivity of the prepared nanocomposite hydrogels. ${ }^{84}$

Moreover, this kind of particles can also be used to provide hydrogels with multiple functionalities beyond the ability to be manipulated through the application of different external stimuli. The intrinsic physicochemical properties of stimuli-responsive 
NPs and/or their manipulation/functionalization with different substances have been exploited in this sense. For example, nanocomposite hydrogels with incorporated MNPs have been evaluated as potential tools for magnetic resonance imaging (MRI). ${ }^{86}$ On the other hand, stimuli-responsive nanofillers were also used as adsorbents of growth factors to then deliver them within hydrogels following the strategies described in Section 2.1. For instance, Zhou et al. decorated graphene oxide flakes with TGF- $\beta 3$, which were then incorporated within collagen hydrogels, allowing the chondrogenic differentiation of the encapsulated hMSCs through the controlled delivery of transforming growth factor. ${ }^{87}$

The combination of the design strategies to prepare nanocomposite hydrogels with stimuli-responsive properties and 3D bioprinting technique has the potential to develop a new generation of advanced scaffolds for TE applications. The modification of bioinks with smart materials that can be stimulated through the application of external stimuli adds a new dimension (time) to the typical 3D bioprinting processes, which have been referred to as "4D bioprinting". 88 The same manufacturing techniques and procedures applied for 3D bioprinting are used when this new "dimension" is incorporated, being the difference in the controlled and predefined functionalities that the smart materials add to the designed composites. ${ }^{89}$ In this manner, the designed 3D bioprinted constructs can be manipulated over time during/after their fabrication by applying different external stimuli, being possible to control their mechanical response, architecture, shape, function, or other physicochemical properties upon exposure to the aforementioned stimuli. The external stimuli applied to induce changes in the physical and/or chemical properties of the smart materials and, consequently, in the constructs where they were incorporated can have different nature, such as heat, light, acoustic waves, $\mathrm{pH}$, or electromagnetic fields.

However, the amount of works that report the incorporation of cells in the (bio)inks and the development of 4 Dbioprinted nanocomposite scaffolds with stimuli-responsive properties is still limited. ${ }^{90}$ For instance, Betsch et al. ${ }^{91}$ exploited the magnetic response of iron oxide NPs incorporated within collagen-agarose bioinks for cartilage TE purposes. A weak magnetic field of $2 \mathrm{mT}$ was applied during the printing process, observing that the unidirectional motion of the MNPs induced by the external stimuli forced the alignment of collagen fibers. Anisotropic composites with random-to-aligned collagen fibers distribution were designed with this strategy, combining aligned layers prepared under the effect of the magnetic field with random layers, mimicking in this way the complex architecture of native cartilage. The designed scaffolds showed reinforced mechanical properties in comparison with monolayer composites and increased the expression of collagen type II of the loaded chondrocytes (Fig. 5a). In a different work from our group, magnetically-responsive cellulose nanocrystals have been incorporated as reinforcement materials in platelet lysate-based bioinks. ${ }^{92}$ The designed bioinks allowed the biofabrication of constructs with high resolution that mimic the fibrillar structure of different native tissues, being also able to support the bioprinting of stem cells with high viability immediately after extrusion and over long-term cell culture. Although external magnetic stimuli was not applied to the 3D bioprinted composites, the ability to magnetically align cellulose nanocrystals within different hydrogels has been widely analyzed in previous works. ${ }^{72,93}$ In this way, the developed bioink is a promising candidate to create 3D bioprinted anisotropic composites with magneto-mechanical stimulation properties through the application of external magnetic fields.

A very interesting design of bioinks with electrically-responsive nanofillers was explored in a recently reported work. ${ }^{94} \mathrm{Ti}_{3} \mathrm{C}_{2} \mathrm{M}$ Xenes nanosheets, novel 2D transition metal carbides/nitrides with outstanding properties in terms of electrical conductivity and biodegradability, were incorporated within hyaluronic acid/ alginate cell-laden inks. The designed bioinks showed high printability and displayed excellent rheological properties, which allowed the fabrication of multilayered scaffolds with high resolution and shape fidelity. Moreover, the incorporation of the nanofillers provided the 3D bioprinted scaffolds with high electrical conductivity, and encapsulated human embryonic kidney 293 cells displayed high viability, suggesting that the designed composites can be applied to engineer electrically responsive tissues. In another relevant example, gold nanowires were incorporated within collagen-based bioinks to provide the printed scaffolds with electrical properties and generate topographical cues for the encapsulated cells. ${ }^{95}$ Although the shear forces generated during the printing process induced a certain orientation of the gold nanostructures, an external electric field of $5 \mathrm{~V}$ and $1 \mathrm{~Hz}$ was applied to accelerate the alignment of the nanofillers within the printed constructs. The designed anisotropic composites induced high alignment degrees of the encapsulated myoblasts and efficient myotube formation, constituting a new prospective platform for the effective formation of muscle tissues (Fig. 5b). Carbon nanotubes have also been evaluated as potential electrically-responsive reinforcement materials for polymeric bioinks. For example, Izadifar et al. ${ }^{96}$ incorporated these nanostructures within alginate-collagen bioinks for cardiac TE applications, observing that the $3 \mathrm{D}$ bioprinted composites offered significantly higher electrical conductivity and a compressive modulus. Moreover, the designed hybrid cardiac constructs promoted the proliferation, migration, and lumen-like organization of the encapsulated human coronary artery endothelial cells, which confirms their great potential for myocardium regeneration and vascularization.

Despite the above-discussed examples, the incorporation of stimuli-responsive materials has a relevant impact on the physicochemical properties and printability of the polymeric solutions, which still requires important hardware configuration evolutions to fully explore all the design possibilities that this technology promises, especially in the case of stimuli-assisted bioprinting. Moreover, the potential cytotoxicity of the nanofillers and/or the applied stimuli over the cells incorporated in the bioinks must also be considered, which still limits the applicability of these 4D bioprinting strategies. ${ }^{17}$

\subsection{Bioinks with nanoparticles for real-time monitoring}

The complexity of 3D bioprinting techniques has been significantly increased in recent years trying to meet the advances in the field of TE. In this sense, the monitoring of different variables, 

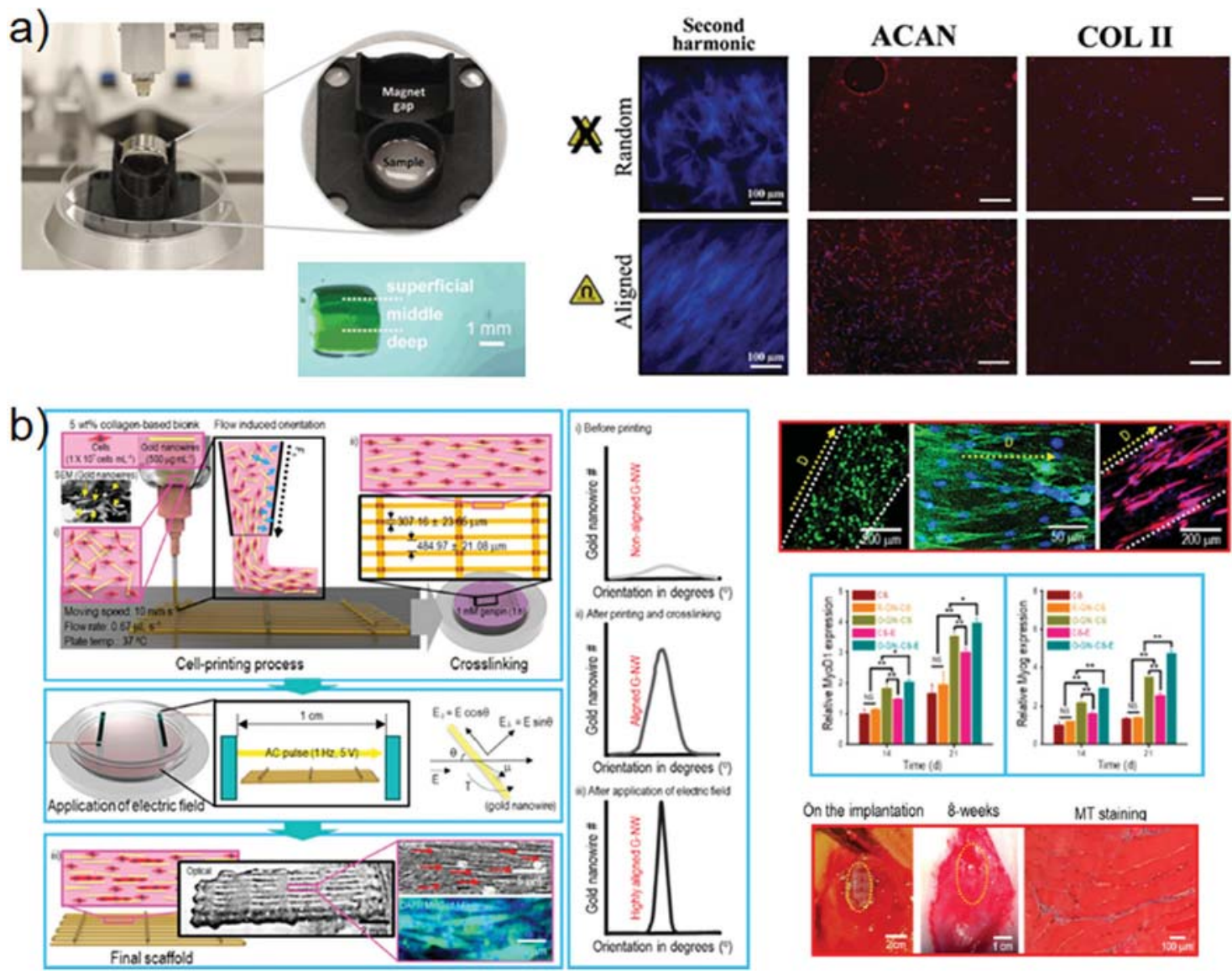

Fig. 5 Bioinks with stimuli-responsive nanoparticles: (a) 3D bioprinting stage including an adaptor for a magnet that allow the application of an external magnetic field during the process (magnetically-assisted bioprinting). This strategy allowed the preparation of bi-layer constructs with random-toaligned collagen fibers organization similar to that observed in native cartilage tissues. In the aligned layer, collagen fibers were organized exploiting the unidirectional traveling motion of the MNPs incorporated in the bioink induced by the applied magnetic field. The chondrocytes embedded in the 3D bioprinted samples produced a matrix with high expressions of ACAN and COL II chondrogenic markers (reproduced with permission from ref. 91 copyright 2018 Wiley-VCH). (b) Schematic illustration of the 3D bioprinting process of collagen-based bioinks modified with gold nanorods for muscle TE: the shear forces generated during the printing induced a relatively high orientation of the nanofillers, which was improved by applying an external electric field to the 3D bioprinted composites. The nanorods acted as cell anchorage sites, enhancing the viability and proliferation of the encapsulated myoblasts. Moreover, the created topographical cues induced significant elongated morphologies and structural alignment of the cells, as well as high expressions of myogenic-related markers. The anisotropic bioprinted composites were also evaluated in vivo through implantations in the temporalis muscle flap, observing their completely transformation into matured myofibers in which nuclei were located on the peripheral region, the final position of the nucleus in muscle maturation process (reproduced with permission from ref. 95 copyright 2019 American Chemical Society).

such as the oxygen gradient or the diffusion of nutrients, has become crucial to ensure the development of real-size 3D bioprinted tissues and organs. ${ }^{97}$ Optical analysis or the use of chemical and physical sensors, among other techniques, have already been explored for this purpose. However, the most recent advancements in 3D cell culture require more sophisticated methods for real-time monitoring based on minimally invasive protocols that ensure negligible effects on cell culture maturation processes. ${ }^{98,99}$ The biochemical indicators are the most effective tools to allow real-time monitoring of the physiological status of healthy and diseased tissues. The progress in this direction can not only ensure precise diagnoses through the development of accurate disease models, but it can also pave the way for effective treatment strategies. ${ }^{98,100}$ In this way, as mentioned in previous sections, the use of NPs with tunable features has been positively evaluated to achieve precise control over the functional and behavioral properties of hydrogel-based systems applied in TE and regenerative medicine. ${ }^{101}$ For instance, Li et al. ${ }^{102}$ developed a hybrid nanocomposite that provided multifunctional properties not only to enhance osteoblastic differentiation and reduce inflammation but also to offer a non-invasive technique to monitor material degradation in vivo through simple fluorescence changes.

Besides optical and biochemical techniques, the integration of electrochemical sensing ${ }^{103}$ in the cell culture of 3D scaffolds to monitor real-time cell environment is another promising direction to develop biomimetic platforms with the capacity to closely observe cell processes. ${ }^{104-106}$ Following this approach, $\mathrm{Hu}$ et al. ${ }^{107}$ combined the biomimetic properties of 3 -aminophenylboronic acid with the mechanical and electrochemical characteristics of a graphene network, thus developing a realtime electrochemical monitoring platform for long-term cell culture. In this way, the design of biomaterials with high 
electroconductivity is established as a promising tool for the electrochemical sensing of cells with high sensitivity and temporalspatial resolution. ${ }^{104,107}$

The modification of bioinks using different NPs has already been explored and proven to be a highly effective strategy to fabricate complex 3D bioprinted constructs, as discussed in previous sections. Currently, in the field of TE, the 3D bioprinting technique is primarily focused on the fabrication of miniature functional equivalents of tissue and/or organs. However, this reduced-scale fabrication approach hinders the monitoring of the 3D constructs, characterized by the presence of multiple cell types and materials, which increase the complexity/variability in the microenvironment created in the confined space. ${ }^{108,109}$ NPS have been proposed as potential tools to monitor the cell maturation within the low-scale 3D bioprinted constructs due to their easy preparation and functionalization, which allow to provide them with the required functionality. ${ }^{110-112}$

Following this approach, bioactive glass NPs were evaluated for the real-time monitoring of 3D printed constructs for bone TEpurposes. ${ }^{113}$ The assembly of poly(citrate-siloxane) on the surface of the NPs increased the toughness of the printed constructs, improved the osteogenic activity, and provided them with photoluminescence, allowing real-time bioimaging monitoring. Similarly, Gou et al. ${ }^{114}$ developed a nanocomposite in which polydiacetylene NPs were dispersed in polymeric matrices for detoxification purposes. The incorporated NPs were able not only to sense the toxins but also to attract and capture them. This work highlights how 3D printed biomimetic nanocomposites can be explored for the generation of detoxification platforms. Although both aforementioned works do not incorporate cells in the $3 \mathrm{D}$ printing process, they provide a valuable proof-ofconcept showing the potential of nanocomposite hydrogels to allow the real-time monitoring of the biofabricated constructs.

So far, the number of works that report the combination of 3D bioprinting strategies with the incorporation of NPs for realtime monitoring purposes is still reduced. For example, NPs functionalized with oxygen-sensitive indicators were developed and incorporated within alginate-methylcellulose-based bioinks in order to monitor the cells encapsulated within the $3 \mathrm{D}$ bioprinted constructs. ${ }^{115}$ Using this system, the oxygen levels and the metabolic activity of different cell types within intact 3D structures were imaged using optical sensors (Fig. 6). This work opens new opportunities to combine the established methods to prepare nanocomposite hydrogels with the development of
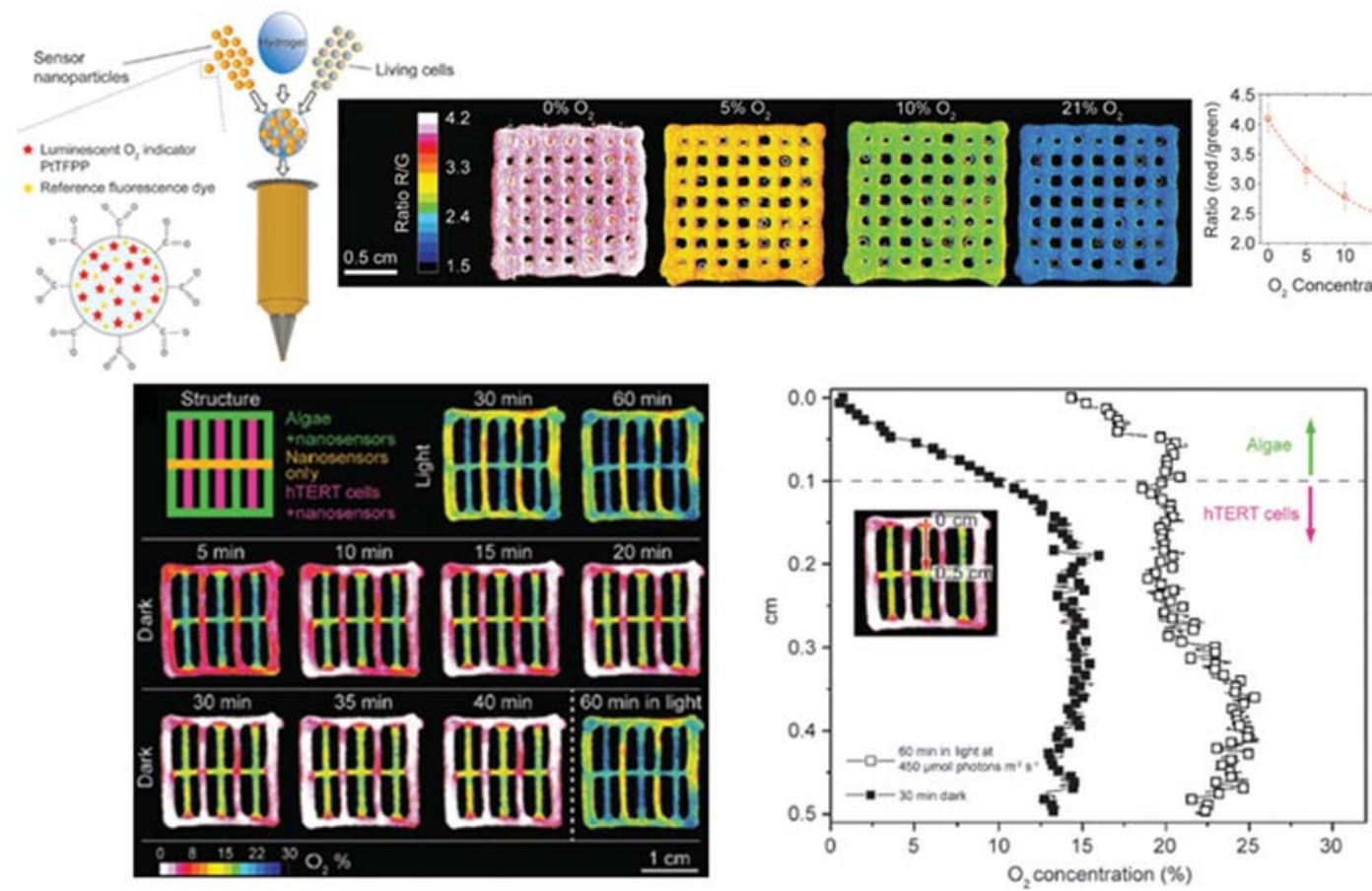

Fig. 6 Bioinks with nanoparticles for real time monitoring: polymeric NPs were modified with an oxygen-sensitive red-emission indicator (platinum(II) meso(2,3,4,5,6-pentafluoro) phenyl porphyrin; PtTFPP) and incorporated within alginate/methylcellulose bioinks. The designed 3D printed scaffolds containing only $\mathrm{O}_{2}$-sensitive NPs (without cells) showed a quasi-exponential decrease in the ratio of $\mathrm{O}_{2}$-dependent luminescence (red) over the constant reference emission (green), with increasing $\mathrm{O}_{2}$ levels ranging from anoxia to full atmospheric saturation $\left(21 \% \mathrm{O}_{2}\right)$. The spatiotemporal dynamics of $\mathrm{O}_{2}$ concentration in multilayered 3D-bioprinted constructs with sensor NPs and green microalgae or human mesenchymal stem cells were also evaluated. The local $\mathrm{O}_{2}$ level was higher in the compartments containing MSCs than in those containing microalgae, indicating mixotrophy of the algal cells with a respiratory activity higher than that of the mammalian cells. The extracted profiles showed the formation of distinct $\mathrm{O}_{2}$ concentration gradients that were affected by the activity of the different cell types and diffusive exchange between the compartments. After 30 min in dark, the $\mathrm{O}_{2}$ concentration in the layer with microalgae reached anoxia about $1 \mathrm{~mm}$ from intersections to layer with MSCs (that only depleted $\mathrm{O}_{2}$ to about $14 \%$ ). In light, the photosynthetic $\mathrm{O}_{2}$ production alleviated the strong $\mathrm{O}_{2}$ depletion in the compartments with microalgae, leading to less $\mathrm{O}_{2}$ concentration differences respect to MSCs layers (reproduced with permission from ref. 115 copyright 2018 Wiley-VCH). 
non-invasive techniques to map cell metabolism and to analyze their microenvironment in real-time after 3D bioprinting.

Despite the few bioprinted constructs that incorporate NPs for monitoring, the developments carried out in this area provide a future prospect to use NPs as biosensing entities in the formulation novel multifunctional bioinks. These new nanocomposite bioinks can be applied to provide the 3D bioprinted tissue/ organs with the capability to accurately monitor cells behavior during long-term culture studies without using invasive techniques. ${ }^{116}$

\section{Concluding remarks and future outlook}

In the last years, 3D bioprinting has emerged as a novel technique with an impressive potential in the biomedical field, enabling the construction of customized complex tissue surrogates with hitherto unknown fidelity and precision. The capability to create spatial patterns with multiple materials and cell types are unique advantages of 3D bioprinting over other fabrication technologies in order to mimic the complexity of human tissues microenvironments. Moreover, the potential of this technique to translate the experiments from micro- to macroscale can help to close the gap between on-bench success to industrial production and clinical translation.

The design of bioinks with the suitable characteristics is an essential factor to provide the final 3D bioprinted constructs with the desired properties for their specific applications. The use of hydrogels is the most common approach to prepare these bioinks, but single-hydrogel based formulations hinder the design of constructs meeting the required properties/ functionalities for the regeneration of certain tissues and for their application in specific interface areas of the biomedical field. In this sense, the incorporation of NPs has emerged as an intriguing choice to develop advanced nanocomposite bioinks with (multi)functional properties. ${ }^{117}$

The different tissues of the human body are characterized by unique microenvironments, making of multiresponsive and multifunctional nanocomposite hydrogel systems ideal candidates to guide a wide range of tissue regeneration processes. Following this concept, the modification of bioink hydrogels with functional nanomaterials has been a logical step to design 3D bioprinted constructs with the desired characteristics for their specific regenerative application. A wide range of NPs has been combined with different biological factors and incorporated within hydrogels to recreate in the bioprinted constructs the complex biological signaling processes that occur in native tissues and organs. Moreover, inorganic NPs, such as calcium phosphates or nanosilicates, have demonstrated their potential to induce the mineralization effects required for the bone or cartilage TE, among other applications. Externally addressable NPs that respond to electromagnetic fields, acoustic waves, or light irradiations have been applied to develop hydrogels with the potential to deliver different stimuli to cells and improved the regeneration of electro-responsive organs/tissues or mechanosensitive tissues. Remarkably, NPs have also been leveraged to develop bioink systems allowing the real-time monitoring of different parameters of cell activity during the in vitro maturation of bioprinted constructs (Table 1 ).

However, the number of works that explored this bioprinting concept is still limited, perhaps due to some unsolved problems related to the addition of nanomaterials to bioink hydrogels. First, the incorporation of functional NPs is an excellent approach to provide the final constructs with desired functionality

Table 1 Main characteristics of different relevant (bio)inks incorporating functional micro- and nanofillers

\begin{tabular}{|c|c|c|c|c|c|c|}
\hline Main functionality & Filler nature & Filler dimensions & (Bio)ink & Cells & Application & Ref. \\
\hline \multirow{3}{*}{$\begin{array}{l}\text { Biological factors } \\
\text { release }\end{array}$} & Gelatin@VEGF particles & $75-125 \mu \mathrm{m}$ & Alginate/matrigel & hEPCs & $\mathrm{TE}$ & 43 \\
\hline & 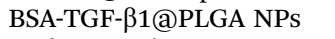 & $60-120 \mathrm{~nm}$ & GelMA/PEGDA & Bone marrow hMSCs & Cartilage TE & 44 \\
\hline & Hydroxyapatite@pDNA NPs & $200 \mathrm{~nm}$ & Alginate/methylcellulose & $\begin{array}{l}\text { Bone marrow-derived } \\
\text { MSCs }\end{array}$ & $\mathrm{TE}$ & 45 \\
\hline \multirow[t]{5}{*}{ Mineralization } & Hydroxyapatite nanorods & $183 \times 34 \mathrm{~nm}$ & $\begin{array}{l}\text { Alginate } \\
\text { Chitosan }\end{array}$ & hASCs & Bone TE & 58 \\
\hline & Hydroxyapatite nanorods & $20 \times 40-60 \mathrm{~nm}$ & Collagen & - & Bone TE & 59 \\
\hline & Hydroxyapatite particles & $12 \mu \mathrm{m}$ & $\begin{array}{l}\text { GelMA } \\
\text { MeHA }\end{array}$ & hASCs & Bone TE & 60 \\
\hline & Hydroxyapatite NPs & $<100 \mathrm{~nm}$ & Collagen & - & Bone TE & 136 \\
\hline & $\begin{array}{l}\text { Nanosilicates } \\
\text { (LAPONITE }^{\circledR} \text { XLG) }\end{array}$ & $1-2 \times 20-50 \mathrm{~nm}$ & GelMA & hMSCs & Bone TE & $\begin{array}{l}68 \text { and } \\
69\end{array}$ \\
\hline Magnetic & Magnetite particles & $20-50 \mu \mathrm{m}$ & Alginate/methylcellulose & hMSCs & Muscoskeletal TE & 90 \\
\hline \multirow[t]{3}{*}{ responsive } & Iron oxide NPs & $10-12 \mathrm{~nm}$ & Collagen/agarose & Chondrocytes & Cartilage TE & 91 \\
\hline & Cellulose nanorods & $206 \times 12 \mathrm{~nm}$ & Platelet lysate & hASCs & $\mathrm{TE}$ & 92 \\
\hline & Iron oxide NPs & $10 \mathrm{~nm}$ & Alginate/methylcellulose & - & - & 132 \\
\hline Electric & $\mathrm{Ti}_{3} \mathrm{C}_{2} \mathrm{MXene}$ nanosheets & $4 \mathrm{~nm} \times 4 \mu \mathrm{m}$ & Alginate/HA & HEK-293 & Neural TE & 94 \\
\hline \multirow[t]{3}{*}{ responsive } & Gold nanowires & - & Collagen & C2C12 myoblasts & Muscle TE & 95 \\
\hline & Carbon nanotubes & $30 \mathrm{~nm} \times 5-20 \mu \mathrm{m}$ & $\begin{array}{l}\text { Alginate } \\
\text { Methacrylated collagen }\end{array}$ & HCAECs & Cardiac TE & 96 \\
\hline & Graphene nanoplatelets & $8 \mathrm{~nm} \times 25 \mu \mathrm{m}$ & Resin & - & Biomedical & 135 \\
\hline $\begin{array}{l}\text { Real-time } \\
\text { monitoring }\end{array}$ & PSMA-PtTFPP NPs & $100-200 \mathrm{~nm}$ & Alginate/methylcellulose & $\begin{array}{l}\text { Microalgae } \\
\text { hTERT-MSCs }\end{array}$ & $\begin{array}{l}\text { Mapping } \mathrm{O}_{2} \\
\text { dynamics }\end{array}$ & 115 \\
\hline
\end{tabular}

PSMA: poly(styrene maleic anhydride); PtTFPP: platinum(II) meso(2,3,4,5,6-pentafluoro)phenyl porphyrin; BSA: bovine serum albumin; PLGA: poly(lactic-co-glycolic acid); pDNA: plasmid DNA; (Me)HA: (methacrylated)hyaluronic acid. 
and tunability, but it also has a considerable impact over the processability of the bioinks. ${ }^{118}$ On the other hand, different types of NPs are characterized by non-negligible cytotoxicity degrees, ${ }^{119,120}$ which limits the amount of particles that can be incorporated in the bioink, making it difficult in cases to provide the final constructs with the desired functionalities. Moreover, the mechanical properties of the majority of 3D bioprinted nanocomposite constructs are still not satisfactory for implementation in certain TE strategies, especially to engineer high-load bearing tissues. The design of 3D bioprinted nanocomposite scaffolding materials displaying the desired functional properties combined with the required mechanical/biological performance is still a challenge. The combination of functional bioinks with structured scaffolds that provide the final hybrid constructs with the desired load-bearing capacity is an interesting and still under explored strategy to address this limitation. In this point, convergence of extrusion or microfluidic-based bioprinting using the described multifunctional bioinks with melt electrowriting or electrospinning technologies emerge as promising approaches to design fibrillar scaffolds with isotropic/anisotropic architectures and controlled load-bearing capacity matching the need of different tissues. ${ }^{121,122}$ Despite the already identified limitations, the remarkable engineering design space that the next generation of 3D bioprinted functional nanocomposites offers for improving the regenerative outcome of a wide range of TE strategies is not easily assessable to alternative technologies, and therefore we expect to see major developments in this field in coming years. Although it is difficult to predict the progress directions of the field, we anticipate that nanocomposite bioinks incorporating NPs combining multiple functionalities will likely be developed. For example, electricallyresponsive NPs decorated with growth factors have already been designed and incorporated within polymeric hydrogels to provide them with stimuli-responsive properties and induce the differentiation of the encapsulated cells. ${ }^{87}$ Moreover, particles that combine electric and magnetic responses have also been synthesized by growing electric shells surrounding MNPs. ${ }^{123}$ These hybrid nanostructures could be particularly useful to prepare 3D bioprinted scaffolds to engineer tissues with anisotropic structure and responsive to electrical stimuli, such as cardiac tissues or nerves. Hybrid ceramic-electric $^{124}$ and ceramic-magnetic ${ }^{125}$ NPs have also been prepared, being an interesting approach to provide 3D bioprinted scaffolds with multifunctional properties in terms of mineralization induction and electromagnetic responsive properties. The incorporation of these nanostructures in the bioinks could be interesting to engineer 3D bioprinted scaffolds for anisotropic tissues with high anisotropic degrees such as cartilage. Moreover, NPs can also be incorporated in the bioinks to provide 3D bioprinted nanocomposites with other functionalities than the main ones that we have discussed in previous sections. For instance, it has been established that silver ${ }^{126}$ and magnesium oxide ${ }^{127}$ NPs show potent antibacterial properties and wound healing capabilities. Similarly, cerium oxide NPs (nanoceria) possess catalytic activity for diverse enzymes and electrochemical characteristics that allow fast electron transfer. ${ }^{128}$ These characteristics have attracted considerable attention in the last few years for the development of highly-sensitive biosensors. ${ }^{128}$ Also, nanoceria has been evaluated as an efficient tool to design nanoplatforms with impressive antioxidant and antimicrobial activities targeting the treatment of diseases characterized by high levels of reactive oxygen species (ROS), among others. ${ }^{129,130}$ For instance, it was demonstrated that polymeric electrospun nanofibers decorated with nanoceria can alleviate ROS production in neonatal primary cardiomyocytes, thus actuating as a negative regulator of agonist-induced cardiac hypertrophy. ${ }^{131} \mathrm{In}$ this way, the incorporation of nanoceria in the bioinks might open multiple possibilities for the fabrication of $3 \mathrm{D}$ bioprinted constructs with multifunctional properties for biomedical purposes.

Beyond the added functionality, nanocomposite bioinks can be leveraged to add a new level of biofabrication control and increase the nano- to macroscale precision of nanocomposite constructs. In this sense, a very interesting underexplored approach is the use of stimuli-assisted bioprinting, which consists of the application of external stimuli to the bioinks during the printing process. This strategy has the potential to significantly increase the control over the design of nanocomposite scaffolding materials with different types of stimuli-responsive NPs. Typically, the responsive properties of the nanofillers incorporated in the bioinks are exploited by applying the specific external stimulus before or after the printing processes. ${ }^{90,132}$ However, in the last few years different works explored the application of the stimuli during the manufacturing step. ${ }^{133}$ This approach is especially appealing in the case of electromagnetic inks, in which the application of external electromagnetic fields during the printing processes (electrically- and magnetically-assisted printing) allows a great control over the spatial distribution of smart nanomaterials and, therefore, over the architecture and anisotropy degree of the printed constructs. For instance, Kokkinis et al. ${ }^{134}$ incorporated a rotary neodymium magnet close to the printing platform of a commercial 3D printer that generates an alternating magnetic field of $40 \mathrm{mT}$ and $8.3 \mathrm{~Hz}$. The drag magnetic forces created by the magnetic setup induced the biaxial alignment of the magnetic materials incorporated in the inks within the plane of the rotating field, designing constructs with well-controlled anisotropy. In a representative recent work in the field of electrically-assisted printing, graphene nanoplatelets were incorporated in polymeric inks and printed under the application of an external electric field of $433 \mathrm{~V} \mathrm{~cm}^{-1}$. The external stimuli applied during the printing process aligned the electrically-responsive nanofillers, creating printed constructs with enhanced toughness and highly-anisotropic electrical properties with potential application in the field of TE. ${ }^{135}$

All these possible bioprinting approaches are just a few examples identified to demonstrate that the potential of nanocomposite bioinks in biomedical applications is wide but still far from being fully exploited. We expect that this perspective review will contribute to foster the discussion on the topic and foresee major developments in bioprinting with nanocomposite bioinks to improve the regenerative outcomes of TE products.

\section{Conflicts of interest}

There are no conflicts to declare. 


\section{Acknowledgements}

Authors acknowledge financial support from the ERC Grant CoG MagTendon nr 772817; FCT - Fundação para a Ciência e a Tecnologia for the PhD grant of SMB (PD/BD/129403/2017), for the contract to MGF (CEECIND/01375/2017); and for project SmarTendon (PTDC/NAN-MAT/30595/2017). AP is grateful to Xunta de Galicia for his postdoctoral grant ED481B2019/025. Some figures were created with BioRender.com.

\section{References}

1 S. Derakhshanfar, R. Mbeleck, K. Xu, X. Zhang, W. Zhong and M. Xing, Bioact. Mater., 2018, 3, 144-156.

2 N. Faramarzi, I. K. Yazdi, M. Nabavinia, A. Gemma, A. Fanelli, A. Caizzone, L. M. Ptaszek, I. Sinha, A. Khademhosseini, J. N. Ruskin and A. Tamayol, Adv. Healthcare Mater., 2018, 7, 1701347.

3 Y. Ding, W. Li, F. Zhang, Z. Liu, N. Zanjanizadeh Ezazi, D. Liu and H. A. Santos, Adv. Funct. Mater., 2019, 29, 1802852.

4 R. Govindan, F. L. Gu, S. Karthi and E. K. Girija, Mater. Today Commun., 2020, 22, 100765.

5 M. Mobaraki, M. Ghaffari, A. Yazdanpanah, Y. Luo and D. K. Mills, Bioprinting, 2020, 18, e00080.

6 J. Groll, J. A. Burdick, D. W. Cho, B. Derby, M. Gelinsky, S. C. Heilshorn, T. Jüngst, J. Malda, V. A. Mironov, K. Nakayama, A. Ovsianikov, W. Sun, S. Takeuchi, J. J. Yoo and T. B. F. Woodfield, Biofabrication, 2019, 11, 013001.

7 A. Schwab, R. Levato, M. D'Este, S. Piluso, D. Eglin and J. Malda, Chem. Rev., 2020, 120, 11028-11055.

8 J. A. Hunt, R. Chen, T. Van Veen and N. Bryan, J. Mater. Chem. B, 2014, 2, 5319-5338.

9 A. Sivashanmugam, R. Arun Kumar, M. Vishnu Priya, S. V. Nair and R. Jayakumar, Eur. Polym. J., 2015, 72, 543-565.

$10 \mathrm{~J}$. H. Lee and H. W. Kim, J. Tissue Eng., 2018, 9, 2041731418768285.

11 M. Gomez-Florit, A. Pardo, R. M. A. Domingues, A. L. Graça, P. S. Babo, R. L. Reis and M. E. Gomes, Molecules, 2020, 25, 5858.

12 A. Pardo, M. Gómez-Florit, S. Barbosa, P. Taboada, R. M. A. Domingues and M. E. Gomes, ACS Nano, 2021, 15, 175-209.

13 H. Zhao, M. Liu, Y. Zhang, J. Yin and R. Pei, Nanoscale, 2020, 12, 14976-14995.

14 N. Asadi, E. Alizadeh, R. Salehi, B. Khalandi, S. Davaran and A. Akbarzadeh, Artif. Cells, Nanomed. Biotechnol., 2018, 46, 465-471.

15 R. Esmaeely Neisiany, M. S. Enayati, P. Sajkiewicz, Z. Pahlevanneshan and S. Ramakrishna, Front. Mater., 2020, 7, 25.

16 S. A. Wilson, L. M. Cross, C. W. Peak and A. K. Gaharwar, ACS Appl. Mater. Interfaces, 2017, 9, 43449-43458.

17 D. Chimene, R. Kaunas and A. K. Gaharwar, Adv. Mater., 2020, 32, 1902026.

18 D. Chimene, K. K. Lennox, R. R. Kaunas and A. K. Gaharwar, Ann. Biomed. Eng., 2016, 44, 2090-2102.
19 T. Ahlfeld, G. Cidonio, D. Kilian, S. Duin, A. R. Akkineni, J. I. Dawson, S. Yang, A. Lode, R. O. C. Oreffo and M. Gelinsky, Biofabrication, 2017, 9, 034103.

20 M. Lee, K. Bae, P. Guillon, J. Chang, Ø. Arlov and M. ZenobiWong, ACS Appl. Mater. Interfaces, 2018, 10, 37820-37828.

21 P. Datta, V. Vyas, S. Dhara, A. R. Chowdhury and A. Barui, J. Bionic Eng., 2019, 16, 842-868.

22 J. H. C. Wang, Q. Guo and B. Li, J. Hand Ther., 2012, 25, 133-141.

23 S. Jana, S. K. L. Levengood and M. Zhang, Adv. Mater., 2016, 28, 10588-10612.

24 G. L. Koons, M. Diba and A. G. Mikos, Nat. Rev. Mater., 2020, 5, 584-603.

25 K. Zhu, S. R. Shin, T. van Kempen, Y. C. Li, V. Ponraj, A. Nasajpour, S. Mandla, N. Hu, X. Liu, J. Leijten, Y. D. Lin, M. A. Hussain, Y. S. Zhang, A. Tamayol and A. Khademhosseini, Adv. Funct. Mater., 2017, 27, 1605352.

26 G. Cidonio, C. R. Alcala-Orozco, K. S. Lim, M. Glinka, I. Mutreja, Y. H. Kim, J. I. Dawson, T. B. F. Woodfield and R. O. C. Oreffo, Biofabrication, 2019, 11(3), 035027, DOI: 10.1088/1758-5090/ab19fd.

27 A. Bhattacharyya, G. Janarthanan and I. Noh, Addit. Manuf., 2021, 37, 101639.

28 X. Du, S. Fu and Y. Zhu, J. Mater. Chem. B, 2018, 6, 4397-4412. 29 J. Simon, E. Flahaut and M. Golzio, Materials, 2019, 12, 624, DOI: 10.3390/ma12040624.

30 S. P. B. Teixeira, R. M. A. Domingues, M. Shevchuk, M. E. Gomes, N. A. Peppas and R. L. Reis, Adv. Funct. Mater., 2020, 30, 1909011.

31 K. Lee, E. A. Silva and D. J. Mooney, J. R. Soc., Interface, 2011, 8, 153-170.

32 Z. Wang, Z. Wang, W. W. Lu, W. Zhen, D. Yang and S. Peng, NPG Asia Mater., 2017, 9, e435.

33 K. Elkhoury, C. S. Russell, L. Sanchez-Gonzalez, A. Mostafavi, T. J. Williams, C. Kahn, N. A. Peppas, E. Arab-Tehrany and A. Tamayol, Adv. Healthcare Mater., 2019, 8, 1900506.

34 R. Eivazzadeh-Keihan, K. K. Chenab, R. Taheri-Ledari, J. Mosafer, S. M. Hashemi, A. Mokhtarzadeh, A. Maleki and M. R. Hamblin, Mater. Sci. Eng., C, 2020, 107, 110267.

35 Y. Lu, W. Sun and Z. Gu, J. Controlled Release, 2014, 194, 1-19.

36 W. He, M. Reaume, M. Hennenfent, B. P. Lee and R. Rajachar, Biomater. Sci., 2020, 8, 3248-3269.

37 Z. Luo, S. Zhang, J. Pan, R. Shi, H. Liu, Y. Lyu, X. Han, Y. Li, Y. Yang, Z. Xu, Y. Sui, E. Luo, Y. Zhang and S. Wei, Biomaterials, 2018, 163, 25-42.

38 M. Fujioka-Kobayashi, M. S. Ota, A. Shimoda, K. I. Nakahama, K. Akiyoshi, Y. Miyamoto and S. Iseki, Biomaterials, 2012, 33, 7613-7620.

39 I. Calejo, R. Costa-Almeida, R. L. Reis and M. E. Gomes, Trends Biotechnol., 2020, 38, 83-98.

40 G. L. Koons and A. G. Mikos, J. Controlled Release, 2019, 295, 50-59.

41 S. Tarafder, A. Koch, Y. Jun, C. Chou, M. R. Awadallah and C. H. Lee, Biofabrication, 2016, 8, 025003.

42 S. Tarafder, J. A. Brito, S. Minhas, L. Effiong, S. Thomopoulos and C. H. Lee, Biofabrication, 2019, 12(1), 015008, DOI: 10.1088/1758-5090/ab48ca. 
43 M. T. Poldervaart, H. Gremmels, K. Van Deventer, J. O. Fledderus, F. C. Öner, M. C. Verhaar, W. J. A. Dhert and J. Alblas, J. Controlled Release, 2014, 184, 58-66.

44 W. Zhu, H. Cui, B. Boualam, F. Masood, E. Flynn, R. D. Rao, Z. Y. Zhang and L. G. Zhang, Nanotechnology, 2018, 29, 185101.

45 T. Gonzalez-Fernandez, S. Rathan, C. Hobbs, P. Pitacco, F. E. Freeman, G. M. Cunniffe, N. J. Dunne, H. O. McCarthy, V. Nicolosi, F. J. O'Brien and D. J. Kelly, J. Controlled Release, 2019, 301, 13-27.

46 J. F. Mano, R. A. Sousa, L. F. Boesel, N. M. Neves and R. L. Reis, Compos. Sci. Technol., 2004, 64, 789-817.

47 K. Gkioni, S. C. G. Leeuwenburgh, T. E. L. Douglas, A. G. Mikos and J. A. Jansen, Tissue Eng., Part B, 2010, 16, 577-585.

48 S. Pina, J. M. Oliveira and R. L. Reis, Adv. Mater., 2015, 27, 1143-1169.

49 A. K. Gaharwar, N. A. Peppas and A. Khademhosseini, Biotechnol. Bioeng., 2014, 111, 441-453.

50 A. M. Brokesh and A. K. Gaharwar, ACS Appl. Mater. Interfaces, 2020, 12, 5319-5344.

51 S. Heid and A. R. Boccaccini, Acta Biomater., 2020, 113, 1-22.

52 X. Wang, E. Tolba, H. C. S. Der, M. Neufurth, Q. Feng, B. R. Diehl-Seifert and W. E. G. Müller, PLoS One, 2014, 9, e112497.

53 D. Tadic and M. Epple, Biomaterials, 2004, 25, 987-994.

54 S. Utech and A. R. Boccaccini, J. Mater. Sci., 2016, 51, 271-310.

55 A. Hoppe, N. S. Güldal and A. R. Boccaccini, Biomaterials, 2011, 32, 2757-2774.

56 H. C. Blair, Q. C. Larrouture, Y. Li, H. Lin, D. Beer-Stoltz, L. Liu, R. S. Tuan, L. J. Robinson, P. H. Schlesinger and D. J. Nelson, Tissue Eng., Part B, 2017, 23, 268-280.

57 X. Wang, H. C. Schröder, M. Wiens, H. Ushijima and W. E. G. Müller, Curr. Opin. Biotechnol., 2012, 23, 570-578.

58 T. T. Demirtaş, G. Irmak and M. Gümüşderelioğlu, Biofabrication, 2017, 9, 035003.

59 G. Montalbano, G. Molino, S. Fiorilli and C. Vitale-Brovarone, J. Eur. Ceram. Soc., 2020, 40, 3689-3697.

60 A. Wenz, K. Borchers, G. E. M. Tovar and P. J. Kluger, Biofabrication, 2017, 9, 044103.

61 Q. Li, X. Lei, X. Wang, Z. Cai, P. Lyu and G. Zhang, Tissue Eng., Part A, 2019, 25, 1261-1271.

62 D. Chimene, D. L. Alge and A. K. Gaharwar, Adv. Mater., 2015, 27, 7261-7284.

63 A. K. Gaharwar, L. M. Cross, C. W. Peak, K. Gold, J. K. Carrow, A. Brokesh and K. A. Singh, Adv. Mater., 2019, 31, 1900332.

64 A. K. Gaharwar, S. M. Mihaila, A. Swami, A. Patel, S. Sant, R. L. Reis, A. P. Marques, M. E. Gomes and A. Khademhosseini, Adv. Mater., 2013, 25, 3329-3336.

65 S. M. Mihaila, A. K. Gaharwar, R. L. Reis, A. Khademhosseini, A. P. Marques and M. E. Gomes, Biomaterials, 2014, 35, 9087-9099.

66 A. Paul, V. Manoharan, D. Krafft, A. Assmann, J. A. Uquillas, S. R. Shin, A. Hasan, M. A. Hussain, A. Memic, A. K. Gaharwar and A. Khademhosseini, J. Mater. Chem. B, 2016, 4, 3544-3554.
67 D. Chimene, C. W. Peak, J. L. Gentry, J. K. Carrow, L. M. Cross, E. Mondragon, G. B. Cardoso, R. Kaunas and A. K. Gaharwar, ACS Appl. Mater. Interfaces, 2018, 10, 9957-9968.

68 D. Chimene, L. Miller, L. M. Cross, M. K. Jaiswal, I. Singh and A. K. Gaharwar, ACS Appl. Mater. Interfaces, 2020, 12, 15976-15988.

69 C. Sears, E. Mondragon, Z. I. Richards, N. Sears, D. Chimene, E. P. McNeill, C. A. Gregory, A. K. Gaharwar and R. Kaunas, Adv. Healthcare Mater., 2020, 9, 1901580, DOI: $10.1002 /$ adhm.201901580.

70 C. R. Alcala-Orozco, I. Mutreja, X. Cui, D. Kumar, G. J. Hooper, K. S. Lim and T. B. F. Woodfield, Bioprinting, 2020, 18, e00073.

71 I. Gholamali, M. Asnaashariisfahani and E. Alipour, Regen. Eng. Transl. Med., 2020, 6, 138-153.

72 M. C. Echave, R. M. A. Domingues, M. Gómez-Florit, J. L. Pedraz, R. L. Reis, G. Orive and M. E. Gomes, ACS Appl. Mater. Interfaces, 2019, 11, 47771-47784.

73 A. Moncion, K. J. Arlotta, E. G. O’Neill, M. Lin, L. A. Mohr, R. T. Franceschi, O. D. Kripfgans, A. J. Putnam and M. L. Fabiilli, Acta Biomater., 2016, 46, 221-233.

74 S. Botelho da Silva, M. Krolicka, L. A. M. van den Broek, A. E. Frissen and C. G. Boeriu, Carbohydr. Polym., 2018, 186, 299-309.

75 K. Ren, Y. Cheng, C. Huang, R. Chen, Z. Wang and J. Wei, J. Mater. Chem. B, 2019, 7, 5704-5712.

76 E. N. Zare, P. Makvandi, B. Ashtari, F. Rossi, A. Motahari and G. Perale, J. Med. Chem., 2020, 63, 1-22.

77 Z. Deng, T. Hu, Q. Lei, J. He, P. X. Ma and B. Guo, ACS Appl. Mater. Interfaces, 2019, 11, 6796-6808.

78 P. Baei, S. Jalili-Firoozinezhad, S. Rajabi-Zeleti, M. TafazzoliShadpour, H. Baharvand and N. Aghdami, Mater. Sci. Eng., C, 2016, 63, 131-141.

79 A. M. Youssef, M. E. A. El-Aziz, E. S. Abd El-Sayed, M. A. Moussa, G. Turky and S. Kamel, Int. J. Biol. Macromol., 2019, 140, 886-894.

80 S. Homaeigohar, T. Y. Tsai, T. H. Young, H. J. Yang and Y. R. Ji, Carbohydr. Polym., 2019, 224, 115112.

81 Y. Xiao and J. Du, J. Mater. Chem. B, 2020, 8, 354-367.

82 A. Pardo, B. Pelaz, J. Gallo, M. Bañobre-López, W. J. Parak, S. Barbosa, P. del Pino and P. Taboada, Chem. Mater., 2020, 32, 2220-2231.

83 J. Mu, C. Hou, H. Wang, Y. Li, Q. Zhang and M. Zhu, Sci. Adv., 2015, 1, e1500533.

84 L. Zhang, X. Zhang, L. Li, Y. Liu, D. Wang, L. Xu, J. Bao and A. Zhang, Macromol. Mater. Eng., 2020, 305, 1900718.

85 A. De Sanctis, J. D. Mehew, M. F. Craciun and S. Russo, Materials, 2018, 11, 1762, DOI: 10.3390/ma11091762.

86 J. Chen, H. Hu, L. Feng, Q. Zhu, A. Hancharou, B. Liu, C. Yan, Y. Xu and R. Guo, Biomed. Mater., 2019, 14, 045013.

87 M. Zhou, N. Lozano, J. K. Wychowaniec, T. Hodgkinson, S. M. Richardson, K. Kostarelos and J. A. Hoyland, Acta Biomater., 2019, 96, 271-280.

88 Y. C. Li, Y. S. Zhang, A. Akpek, S. R. Shin and A. Khademhosseini, Biofabrication, 2017, 9, 012001.

89 D. G. Tamay, T. D. Usal, A. S. Alagoz, D. Yucel, N. Hasirci and V. Hasirci, Front. Bioeng. Biotechnol., 2019, 7, 164. 
90 J. Spangenberg, D. Kilian, C. Czichy, T. Ahlfeld, A. Lode, S. Günther, S. Odenbach and M. Gelinsky, ACS Biomater. Sci. Eng., 2021, 7(2), 648-662.

91 M. Betsch, C. Cristian, Y. Y. Lin, A. Blaeser, J. Schöneberg, M. Vogt, E. M. Buhl, H. Fischer and D. F. Duarte Campos, Adv. Healthcare Mater., 2018, 7, 1800894.

92 B. B. Mendes, M. Gómez-Florit, A. G. Hamilton, M. S. Detamore, R. M. A. Domingues, R. L. Reis and M. E. Gomes, Biofabrication, 2020, 12, 015012.

93 K. J. De France, K. G. Yager, K. J. W. Chan, B. Corbett, E. D. Cranston and T. Hoare, Nano Lett., 2017, 17, 6487-6495.

94 H. Rastin, B. Zhang, A. Mazinani, K. Hassan, J. Bi, T. T. Tung and D. Losic, Nanoscale, 2020, 12, 16069-16080.

95 W. J. Kim, C. H. Jang and G. H. Kim, Nano Lett., 2019, 19, 8612-8620.

96 M. Izadifar, D. Chapman, P. Babyn, X. Chen and M. E. Kelly, Tissue Eng., Part C, 2018, 24, 74-88.

97 B. Gao, Q. Yang, X. Zhao, G. Jin, Y. Ma and F. Xu, Trends Biotechnol., 2016, 34, 746-756.

98 X. Liu, H. Yuk, S. Lin, G. A. Parada, T.-C. Tang, E. Tham, C. de la Fuente-Nunez, T. K. Lu and X. Zhao, Adv. Mater., 2018, 30, 1704821.

99 Z. Tang, Y. Wang, P. Podsiadlo and N. A. Kotov, Adv. Mater., 2006, 18, 3203-3224.

100 Y. Pan, N. Hu, X. Wei, L. Gong, B. Zhang, H. Wan and P. Wang, Biosens. Bioelectron., 2019, 130, 344-351.

101 L. K. Bogart, G. Pourroy, C. J. Murphy, V. Puntes, T. Pellegrino, D. Rosenblum, D. Peer and R. Lévy, ACS Nano, 2014, 8, 3107-3122.

102 Y. Li, Y. Guo, W. Niu, M. Chen, Y. Xue, J. Ge, P. X. Ma and B. Lei, ACS Appl. Mater. Interfaces, 2018, 10, 17722-17731.

103 J. Kieninger, A. Weltin, H. Flamm and G. A. Urban, Lab Chip, 2018, 18, 1274-1291.

104 K. Hiramoto, K. Ino, Y. Nashimoto, K. Ito and H. Shiku, Front. Chem., 2019, 7, 396.

105 S. Andreescu and O. A. Sadik, Methods, 2005, 37, 84-93.

106 M. M. Liu, Z. Z. Guo, H. Liu, S. H. Li, Y. Chen, Y. Zhong, Y. Lei, X. H. Lin and A. L. Liu, Biosens. Bioelectron., 2019, 144, 111686.

107 X. B. Hu, Y. L. Liu, W. J. Wang, H. W. Zhang, Y. Qin, S. Guo, X. W. Zhang, L. Fu and W. H. Huang, Anal. Chem., 2018, 90, 1136-1141.

108 M. Castilho, M. de Ruijter, S. Beirne, C. C. Villette, K. Ito, G. G. Wallace and J. Malda, Trends Biotechnol., 2020, 38(12), 1316-1328.

109 H.-G. Yi, H. Lee and D.-W. Cho, Bioengineering, 2017, 4, 10.

110 Y. Lu, A. A. Aimetti, R. Langer and Z. Gu, Nat. Rev. Mater., 2017, 2, 16075.

111 C. Aimé and T. Coradin, J. Polym. Sci., Part B: Polym. Phys., 2012, 50, 669-680.

112 F. Fiorini, E. A. Prasetyanto, F. Taraballi, L. Pandolfi, F. Monroy, I. López-Montero, E. Tasciotti and L. De Cola, Small, 2016, 12, 4881-4893.
113 M. Chen, F. Zhao, Y. Li, M. Wang, X. Chen and B. Lei, Mater. Sci. Eng., C, 2020, 106, 110153, DOI: 10.1016/j.msec.2019.110153.

114 M. Gou, X. Qu, W. Zhu, M. Xiang, J. Yang, K. Zhang, Y. Wei and S. Chen, Nat. Commun., 2014, 5, 1-9.

115 E. Trampe, K. Koren, A. R. Akkineni, C. Senwitz, F. Krujatz, A. Lode, M. Gelinsky and M. Kühl, Adv. Funct. Mater., 2018, 28, 1804411.

116 L. K. Narayanan, T. L. Thompson, A. Bhat, B. Starly and R. A. Shirwaiker, ASME Int., 2017, V004T05A013.

117 J. Göhl, K. Markstedt, A. Mark, K. Håkansson, P. Gatenholm and F. Edelvik, Biofabrication, 2018, 10(3), 034105, DOI: 10.1088/1758-5090/aac872.

118 S. Ribeiro, T. Ribeiro, C. Ribeiro, D. M. Correia, J. P. S. Farinha, A. C. Gomes, C. Baleizão and S. Lanceros-Méndez, Nanomaterials, 2018, 8, 933, DOI: 10.20944/PREPRINTS 201810.0598.V1.

119 Z. Jiang, K. Shan, J. Song, J. Liu, S. Rajendran, A. Pugazhendhi, J. A. Jacob and B. Chen, Life Sci., 2019, 220, 156-161.

120 Y. P. Jia, B. Y. Ma, X. W. Wei and Z. Y. Qian, Chin. Chem. Lett., 2017, 28, 691-702.

121 J. Xue, T. Wu, Y. Dai and Y. Xia, Chem. Rev., 2019, 119, 5298-5415.

122 M. Castilho, M. de Ruijter, S. Beirne, C. C. Villette, K. Ito, G. G. Wallace and J. Malda, Trends Biotechnol., 2020, 38, 1316-1328.

123 C. M. Ramírez-acosta, J. Cifuentes, M. C. Castellanos, R. J. Moreno, C. Muñoz-camargo, J. C. Cruz and L. H. Reyes, Pharmaceutics, 2020, 12, 1-25.

124 H. Liang, X. Xu, X. Feng, L. Ma, X. Deng, S. Wu, X. Liu and C. Yang, Int. J. Nanomed., 2019, 14, 6151-6163.

125 A. Seyfoori, S. A. S. Ebrahimi, S. Omidian and S. M. Naghib, J. Taiwan Inst. Chem. Eng., 2019, 96, 503-508.

126 E. Marsich, F. Bellomo, G. Turco, A. Travan, I. Donati and S. Paoletti, J. Mater. Sci.: Mater. Med., 2013, 24, 1799-1807.

127 P. K. Stoimenov, R. L. Klinger, G. L. Marchin and K. J. Klabunde, Langmuir, 2002, 18, 6679-6686.

128 F. Charbgoo, M. Ramezani and M. Darroudi, Biosens. Bioelectron., 2017, 96, 33-43.

129 A. Dhall and W. Self, Antioxidants, 2018, 7, 97.

130 S. Rajeshkumar and P. Naik, Biotechnol. Rep., 2018, 17, 1-5. 131 A. Jain, M. Behera, C. Mahapatra, N. R. Sundaresan and K. Chatterjee, Mater. Sci. Eng., C, 2021, 118, 111416.

132 D. Podstawczyk, M. Nizioł, P. Szymczyk, P. Wiśniewski and A. Guiseppi-Elie, Addit. Manuf., 2020, 34, 101275.

133 Y. Yang, Z. Chen, X. Song, Z. Zhang, J. Zhang, K. K. Shung, Q. Zhou and Y. Chen, Adv. Mater., 2017, 29, 1605750.

134 D. Kokkinis, M. Schaffner and A. R. Studart, Nat. Commun., 2015, 6, 8643.

135 Y. Yang, X. Li, M. Chu, H. Sun, J. Jin, K. Yu, Q. Wang, Q. Zhou and Y. Chen, Sci. Adv., 2019, 5, eaau9490.

136 Q. Li, X. Lei, X. Wang, Z. Cai, P. Lyu and G. Zhang, Tissue Eng., Part A, 2019, 25, 1261-1271. 